\title{
Hot subdwarf B stars with neutron star components
}

\section{Binary population synthesis}

\author{
You $\mathrm{Wu}^{1,2,3}$, Xuefei Chen ${ }^{1,2,4}$, Hailiang Chen ${ }^{1,2,4}$, Zhenwei $\mathrm{Li}^{1,2,3}$, and Zhanwen $\operatorname{Han}^{1,2,4}$ \\ 1 Yunnan Observatories, Chinese Academy of Sciences, Kunming 650216, PR China \\ e-mail: youwu@ynao.ac.cn, cxf@ynao.ac.cn \\ 2 Key Laboratory for the Structure and Evolution of Celestial Objects, Chinese Academy of Sciences, Kunming 650216, PR China \\ 3 University of the Chinese Academy of Science, Beijing 100049, PR China \\ ${ }^{4}$ Center for Astronomical Mega-Science, Chinese Academy of Sciences, 20A Datun Road, Chaoyang District, Beijing 100012, \\ PR China
}

Received 27 April 2019 / Accepted 1 December 2019

\begin{abstract}
Context. Subdwarf B stars (sdBs) play a crucial role in stellar evolution, asteroseismology, and far-UV radiation of early-type galaxies, and have been intensively studied with observation and theory. It has theoretically been predicted that sdBs with neutron star (NS) companions exist in the Galaxy, but none have been discovered yet. This remains a puzzle in this field. In a previous study (hereafter Paper I), we have studied the formation channels of sdB+NS binaries from main-sequence (MS) stars plus NS binaries by establishing a model grid, but it is still unclear how these binaries consisting of MS stars and NS binaries came to be in the first place.

Aims. We systematically study the formation of sdB+NS binaries from their original zero-age main-sequence progenitors. We bridge the gap left by our previous study in this way. We obtain the statistical population properties of sdB+NS binaries and provide some guidance for observational efforts.

Methods. We first used Hurley's rapid binary evolution code BSE to evolve $10^{7}$ primordial binaries to the point where the companions of NS+MS, NS+Hertzsprung gap star, and NS+Giant Branch star binaries have just filled their Roche lobes. Next, we injected these binaries into the model grid we developed in Paper I to obtain the properties of the sdB+NS populations. We adopted two prescriptions of NS natal kicks: the classical Maxwellian distribution with a dispersion of $\sigma=265 \mathrm{~km} \mathrm{~s}^{-1}$, and a linear formula that assumes that the kick velocity is associated with the ratio of ejected to remnant mass. Different values of $\alpha_{\mathrm{CE}}$, where $\alpha_{\mathrm{CE}}$ is the common-envelope ejection efficiency, were chosen to examine the effect of common-envelope evolution on the results.

Results. In the Galaxy, the birthrate of $\mathrm{sdB}+\mathrm{NS}$ binaries is about $10^{-4} \mathrm{yr}^{-1}$ and there are $\sim 7000-21000$ such binaries. This contributes $0.3-0.5 \%$ of all sdB binaries in the most favorable case. Most Galactic sdB $+\mathrm{NS}$ binaries $(\gtrsim 60 \%)$ arise from the channel of stable mass transfer. The value of $\alpha_{\mathrm{CE}}$ has little effect on the results, but when we use the linear formula prescription of NS natal kick, the number and birthrate doubles in comparison to the results we obtained with the Maxwellian distribution. The orbital periods of sdB+NS binaries from different formation channels differ significantly, as expected. This results in two peaks in the radial velocity (RV) semi-amplitude distribution: $100-150 \mathrm{~km} \mathrm{~s}^{-1}$ for stable mass transfer, and $400-600 \mathrm{~km} \mathrm{~s}^{-1}$ for common-envelope ejection. However, the two $\mathrm{sdB}+\mathrm{NS}$ binary populations exhibit similar delay-time distributions, which both peak at about $0.2 \mathrm{Gyr}$. This indicates that Galactic $\mathrm{sdB}+\mathrm{NS}$ binaries are born in very young populations, probably in the Galactic disk. The sdB+NS binaries produced from the common-envelope ejection channel are potential sources of strong gravitational wave radiation (GWR), and about $\sim 100-300$ could be detected by the Laser Interferometer Space Antenna (LISA) with a signal-to-noise ratio of 1.

Conclusions. Most sdB+NS binaries are located in the Galactic disk with small RV semi-amplitudes. SdB+NS binaries with large RV semi-amplitudes are expected to be strong GWR sources, some of which could be detected by LISA in the future.
\end{abstract}

Key words. binaries: general - stars: neutron - subdwarfs - stars: formation

\section{Introduction}

Hot subdwarf B (sdB) stars, also known as extreme horizontal branch stars, are He-core burning stars with very thin hydrogenrich envelopes $\left(\lesssim 0.02 M_{\odot}\right)$ (Heber 1986$)$. These objects exhibit high effective temperatures $\left(T_{\text {eff }} \approx 20000-40000 \mathrm{~K}\right)$ and high surface gravities $\left(\log g\left(\mathrm{~cm} \mathrm{~s}^{-2}\right) \sim 5.0-6.5\right)$ (Heber 2016). SdBs play a crucial role in stellar evolution and asteroseismology and heavily influence the far-UV radiation of early-type galaxies (Charpinet et al. 1996, 1997; Kilkenny et al. 1997; Brown et al. 2000; Han et al. 2007). They have consequently attracted intensive interest from observers and theorists alike (e.g., Han et al. 2002, 2003; Geier et al. 2010; Chen et al. 2013; Vos et al. 2018. A large portion of sdBs are binaries (Maxted et al. 2001; Napiwotzki et al. 2004). Han et al. (2002, 2003) developed a binary model for the formation of sdBs, in which sdB binaries originate from either stable Roche-lobe overflow (the RLOF channel) or from common-envelope ejection (the CE channel), while single sdBs arise from mergers of two He white dwarfs (WDs) (see also Webbink 1984). The model nicely reproduced properties of short-period sdB+WD binaries and single sdBs. Following the discovery of sdB binaries with long orbital periods (Deca et al. 2012), Chen et al. (2013) revisited the period distribution by considering the mass-luminosity relation of the cores of giant stars. This period distribution has been confirmed by recent studies (Vos et al. 2019).

We here study sdBs with neutron star (NS) or black hole (BH) companions. Theoretically, these objects can be produced through binary evolution, and they may eventually evolve to become $\mathrm{WD}+\mathrm{NS}$ or $\mathrm{WD}+\mathrm{BH}$ binaries. For example, $\mathrm{sdB}+\mathrm{NS}$ binaries 
can be produced from the evolution of systems that eventually become HeCO-WD+NS binaries (Podsiadlowski et al. 2002). Tauris et al. (2011) also clearly demonstrated the existence of an intermediate evolutionary phase of $\mathrm{sdB}+\mathrm{NS}$ binaries when they modeled the formation of the massive millisecond-pulsar binary PSR J1614-2230. Nelemans (2010) performed binary population synthesis (BPS) studies for sdBs and predicted that about $1 \%$ of sdBs in the Galaxy have NS companions and $0.1 \%$ have BH companions. They considered the effect of asymmetric kicks that are imparted on NSs by their host supernovae, but ignored the details of the formation process of $\mathrm{sdB}+\mathrm{NS}$ binaries.

Geier et al. (2011) proposed the project Massive Unseen Companions to Hot Faint Under-luminous Stars from SDSS (MUCHFUSS), with the aim of finding massive compact companions, such as massive WDs $\left(>1.0 M_{\odot}\right)$, NSs, or stellar mass $\mathrm{BHs}$, of hot $\mathrm{sdB} / \mathrm{O}$ stars. So far, they have detected 53 candidates in total, but did not find any evidence for the existence of NS or BH companions to sdB/O stars (Geier et al. 2015, 2017). They proceeded to suggest $1.5 \%$ as an upper limit for the fraction of close $\mathrm{sdB}+\mathrm{NS}$ or $\mathrm{sdB}+\mathrm{BH}$ binaries.

In order to better understand the observations of MUCHFUSS, Wu et al. (2018; hereinafter Paper I) systematically investigated the formation of $\mathrm{sdB}+\mathrm{NS}$ binaries by establishing a model grid for a series of main-sequence star (MS)+NS binaries. They showed that $\mathrm{sdB}+\mathrm{NS}$ binaries may be produced either from the RLOF channel or from the CE channel. For those that evolved through the RLOF channel, the orbital period ranges from several days to more than 1000 days. The highest radial velocity (RV) semi-amplitude $K$ is about $150 \mathrm{~km} \mathrm{~s}^{-1}$. For those from the CE channel, the orbital period is very short, with high values of $K$ of up to $800 \mathrm{~km} \mathrm{~s}^{-1}$. Gravitational wave radiation (GWR) may cause them to merge on a timescale of only some megayears. This implies that $\mathrm{sdB}+\mathrm{NS}$ binaries from both channels are difficult to discover.

The main goal of this study is to obtain the properties of Galactic sdB+NS binaries, such as the number, period distribution, dependence on the age, and the uncertainties of binary evolution and NS natal kicks, by combining a new BPS study with the model grid established in Paper I. The remaining part of the paper is structured as follows. In Sect. 2 we present the formation channels of $\mathrm{sdB}+\mathrm{NS}$ binaries from primordial binaries. Section 3 contains the methods we used in our BPS simulation and the parameter settings of our binary evolution. The simulation results are shown in Sect. 4, and the main conclusions are summarized in Sect. 5.

\section{Formation scenario for $\mathbf{s d B}+\mathrm{NS}$ binaries}

Figure 1 illustrates the evolution of a primordial binary into an $\mathrm{sdB}+\mathrm{NS}$ binary. The primary (the initially more massive component) evolves faster and fills its Roche lobe first, then transfers material to the secondary. The mass transfer can be dynamically stable or unstable, depending on the initial mass ratio. For binaries with stable mass transfer, the companion star (i.e., the initially less massive component) is relatively massive in comparison to its counterparts in systems with dynamically unstable mass transfer because of the constraint of the mass ratio for stable mass transfer. They subsequently accrete some of the material during the mass-transfer phase, which increases their mass. Subsequently, the produced NS+MS binaries have relatively large MS companions, that is, the MS mass $\gtrsim 10 M_{\odot}$ Because of the high mass ratio of these binaries, they ultimately undergo unstable mass transfer and enter the $\mathrm{CE}$ phase when the secondary fills its Roche lobe. If the binaries survive this CE phase, the secondaries evolve into massive He stars with very high effective temperatures. These are not sdB stars.

For the case of dynamically unstable mass transfer, the binary enters a CE phase and evolves into a He+MS binary after the ejection of its $\mathrm{CE}$. The He star further evolves and leaves behind an NS after supernova explosion if its mass is in the appropriate range. The secondary (the present MS star) evolves and subsequently fills its Roche lobe. What happens next depends upon the mass of the secondary and the orbital period. The mass transfer may be dynamically stable or unstable (to form a $\mathrm{CE}$ ). Both cases may produce $\mathrm{sdB}+\mathrm{NS}$ binaries, according to the results of Paper I (and this is referred to here as the $\mathrm{CE}+\mathrm{CE}$ channel and CE+RLOF channel, respectively). In our calculations, we assume that the system forms an $\mathrm{sdB}$ if helium can be ignited successfully, steady burning in the center of stars is maintained after the mass-transfer process, the envelope is stripped to such an extent that its mass is relatively low, and if the effective temperature is lower than $4 \times 10^{4} \mathrm{~K}$.

By investigating the evolution of a series of NS+MS binaries with the MESA code (Paxton et al. 2011, 2013, 2015), we obtained the parameter space for producing $\mathrm{sdB}+\mathrm{NS}$ binaries through the RLOF and CE channels in Paper I, as shown in the companion mass - orbit period $\left(M_{2}-\lg P_{\mathrm{m}}\right)$ diagram of Fig. 2, where $M_{2}$ is the secondary mass and $P_{\mathrm{m}}$ is the orbital period when the secondary fills its Roche lobe. In the calculation, we followed the detailed evolution of the mass-transfer rate for each binary. In systems where the mass-transfer rate increases dramatically in a short time and becomes higher than a critical rate, that is, $10^{-4} M_{\odot} \mathrm{yr}^{-1}$, we assume that the binary system undergoes dynamically unstable mass transfer.

In the CE channel, we adopt the standard energy prescription for CE evolution (see Sect. 3.3 for details). The binding energy here considers the combined effects of gravitational energy and internal energy and is slightly different from that of Paper I, where these two sources of energy were considered separately (see their Eq. (1)). In Paper I, we used a different approach for calculating the $\mathrm{CE}$ evolution, therefore this leads to an inconsistency in the treatment of the two CE phases in the present study. To resolve this problem, we repeated the CE evolution in Paper I, adopting the same prescriptions for the CE that are used in this study, including the calculation of $\lambda$ and different $\alpha_{\mathrm{CE}}$ values, and substitute the results of Paper I with those of this new set of calculations.

The CE channel and the stable RLOF channel in Fig. 2 are separated by a gap. When the orbital periods of the NS+MS, $\mathrm{NS}+$ Hertzsprung gap (HG), and NS+Giant Branch (GB) binaries lie in this gap, the mass-transfer process is dynamically unstable and a CE forms, but the CE cannot be ejected successfully because its binding energy is high. The parameter space lies in a fairly narrow range when the mass of the companion star is lower than $2.5 M_{\odot}$ : He can only be ignited subsequently when the donors start mass transfer close to the tip of the red giant branch if the donors have degenerate He cores. There is a transition from degenerate to non-degenerate $\mathrm{He}$ cores when the mass of the companion stars lies in the range of $2.0-2.5 M_{\odot}$, resulting in a small parameter space for producing sdBs in this mass range.

\section{Simulation of the binary population synthesis}

In order to obtain the properties of Galactic sdB+NS binaries, such as the number, period distribution, dependence on the age or metallicity, we performed eight sets of BPS simulations. For each simulation set, we generated $10^{7}$ primordial binaries by a Monte Carlo method, and then we used the rapid binary stellar 


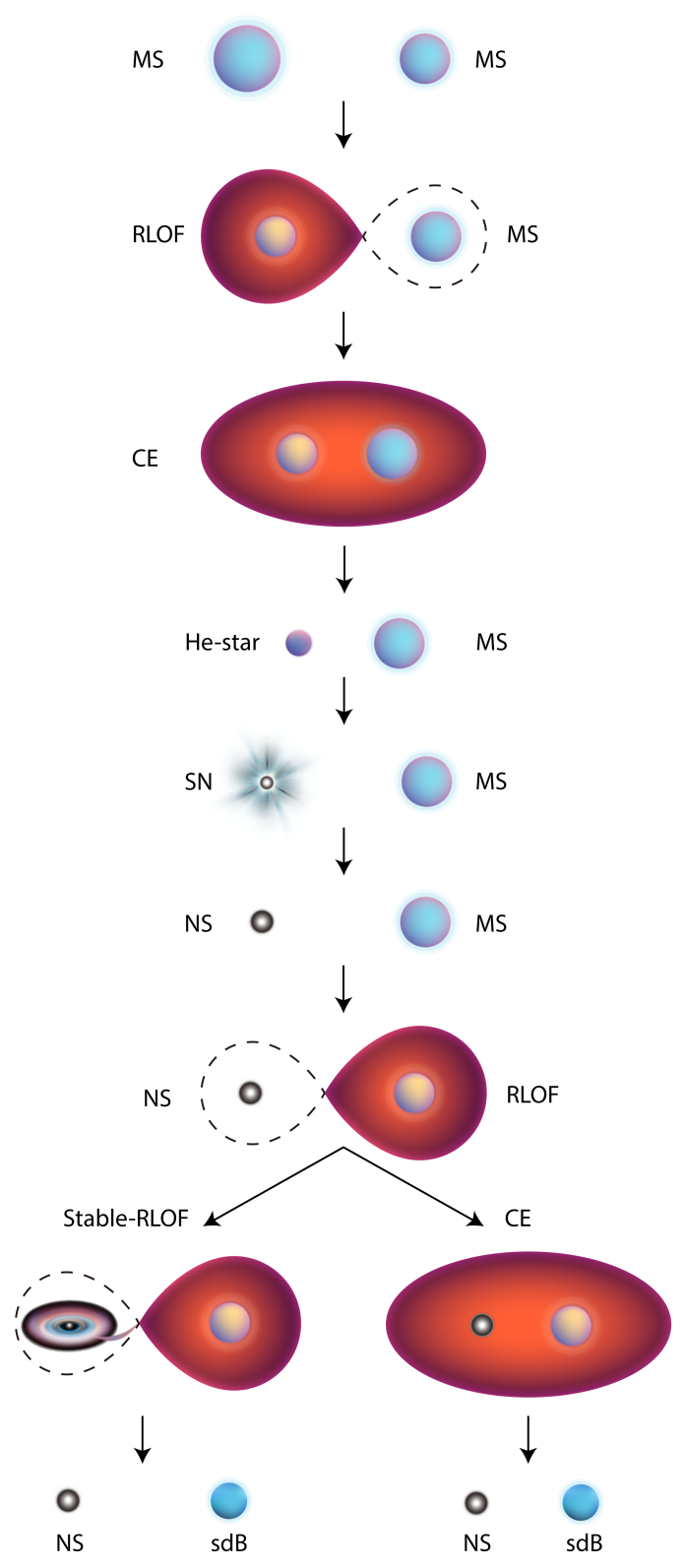

Fig. 1. Illustration of the formation of $s d B+N S$ binaries. For the first mass-transfer process, we do not show the stable-RLOF case in the figure because the NS+MS binaries produced by stable RLOF do not contribute to the production of sdB+NS binaries (see Sect. 2 for details).

evolution code BSE (Hurley et al. 2000, 2002) to evolve these binaries to obtain a sample of binaries consisting of an NS and a non-degenerate companion (i.e., MS, HG, and GB), in which the companion stars just fill their Roche lobes and start transferring mass to the NSs. We use $M_{2}$ to denote the companion mass hereinafter for convenience. We then combined these binaries with the model grid and obtained the information for the $\mathrm{sdB}+\mathrm{NS}$ binaries.

We study Population I stars with a metallicity of $Z=0.02$. The physics inputs for the eight sets are summarized in Table 1, and the details are described below.

\subsection{Initial distribution for binary parameters}

To generate the primordial binaries, we need the initial distributions for the binary parameters as inputs in the Monte Carlo

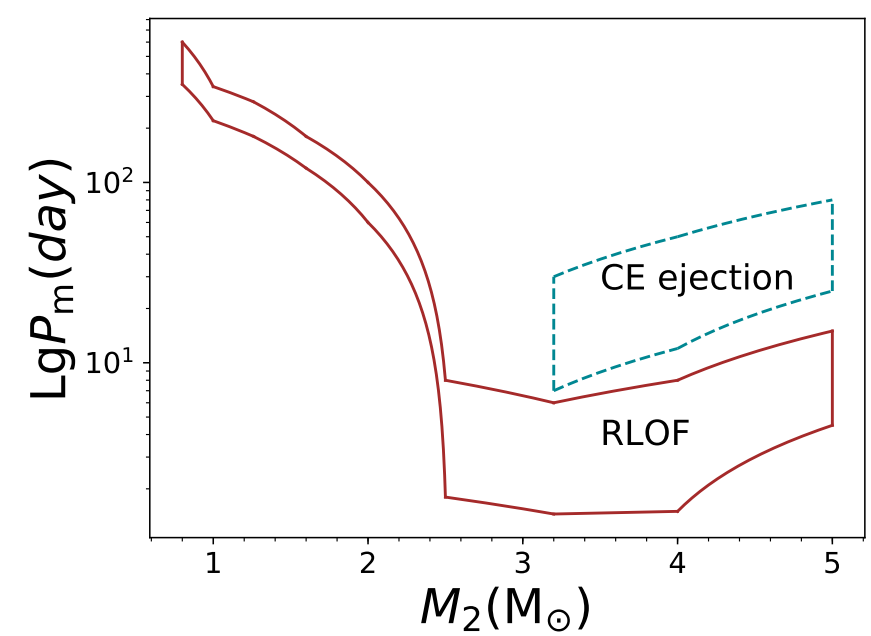

Fig. 2. Parameter spaces for NS+MS, NS+HG, and NS+GB binaries that can produce $\mathrm{sdB}+\mathrm{NS}$ binaries, where $M_{2}$ is the companion mass and $P_{\mathrm{m}}$ is the orbital period when the companion fills its Roche lobe. $M_{2}$ ranges from $0.8 M_{\odot}$ to $5.0 M_{\odot}$ (MS stars with mass $>5.0 M_{\odot}$ could soon evolve to high-mass X-ray binaries through accretion by the stellar wind). The regions enclosed with the solid and dashed lines are for the stable RLOF channel and the CE ejection channel, respectively. In the CE channel, $\alpha_{\mathrm{CE}}=1$. The results for $\alpha_{\mathrm{CE}}=0.5,2$, and 3 are shown in Fig. 3 .

Table 1. Parameters for the BPS simulation in our study.

\begin{tabular}{ccc}
\hline \hline Set & Natal kick & $\alpha_{\mathrm{CE}}$ \\
\hline 1 & $V k_{\text {linear }}$ & 0.5 \\
2 & $V k_{\text {linear }}$ & 1 \\
3 & $V k_{\text {linear }}$ & 2 \\
4 & $V k_{\text {linear }}$ & 3 \\
5 & $V k_{\sigma=265 \mathrm{~km} \mathrm{~s}^{-1}}$ & 0.5 \\
6 & $V k_{\sigma=265 \mathrm{~km} \mathrm{~s}^{-1}}$ & 1 \\
7 & $V k_{\sigma=265 \mathrm{~km} \mathrm{~s}^{-1}}$ & 2 \\
8 & $V k_{\sigma=265 \mathrm{~km} \mathrm{~s}^{-1}}$ & 3 \\
\hline
\end{tabular}

Notes. $V k_{\text {linear }}$ and $V k_{\sigma=265 \mathrm{~km} \mathrm{~s}^{-1}}$ are for the prescriptions of NS natal kicks from CCSNe, i.e., Eqs. (3) and (4), respectively. $\alpha_{\mathrm{CE}}$ is the CE ejection efficiency.

simulation, that is, primary mass, mass ratio, and orbital separation. We describe these as follows.

(i) We adopted the initial mass function of Miller \& Scalo (1979) for the distribution of the primary mass $M_{1}$, as given by Eggleton et al. (1989),

$M_{1}=\frac{0.19 X}{(1-X)^{0.75}+0.032(1-X)^{1 / 4}}$,

where $X$ is a random number in the range from 0 to 1 .

(ii) The initial mass-ratio $q$ (The ratio of the primary to the secondary) distribution is taken as a constant distribution, that is, $n(1 / q)=1$.

(iii) The distribution of the initial separation is a uniform distribution in $\log a$ for wide binaries and a power-law distribution at close separation (Han 1998),

$a n(a)=\left\{\begin{array}{c}\alpha_{\mathrm{sep}}\left(\frac{a}{a_{0}}\right)^{m}, \quad a \leq a_{0} ; \\ \alpha_{\mathrm{sep}}, \quad a_{0}<a<a_{1},\end{array}\right.$

where $a$ is orbital separation, $\alpha_{\text {sep }} \approx 0.07, a_{0}=10 R_{\odot}, a_{1}=5.75 \times$ $10^{6} R_{\odot}$, and $m \approx 1.2$. This distribution yields approximately $50 \%$ of the binaries with orbital periods shorter than $100 \mathrm{yr}$. 


\subsection{NS formation and their kicks}

It is known that NSs may be produced from core-collapse supernovae (CCSNe), electron-capture supernovae (ECSNe) (Miyaji et al. 1980), or accretion-induced collapse (AIC) of an oxygen-neon-magnesium WD (ONeMg WD) (Nomoto 1984, 1987; Michel 1987). In our study, the CCSNe occurs for a star with a He core mass $M_{\mathrm{He}}>2.25 M_{\odot}$, leaving an NS or a black hole after supernovae. When the remnant mass (for the calculation of the remnant mass, we refer to Hurley et al. 2000) was lower than $3.0 M_{\odot}$, we assumed that an NS was left (according to the Tolman-Oppenheimer-Volkoff limit in Oppenheimer \& Volkoff 1939). Similar to Chruslinska et al. (2018), we assumed that a star with a He-core mass in the range of $1.83 M_{\odot}<M_{\mathrm{He}}<2.25 M_{\odot}$ produces an NS from ECSNe when the remnant mass approaches the Chandrasekhar mass limit. In the AIC formation scenario, the mass accumulation efficiency of the WDs was computed as follows. When the mass-transfer rate exceeded a critical mass-transfer rate, we employed the optically thick wind model proposed by Hachisu et al. (1996). In the stable burning region of $\mathrm{H}$ or $\mathrm{He}$, the mass-accumulation efficiency was assumed to be 1 . When the mass-transfer rate was lower than the minimum accretion rate of stable burning, we separately computed the mass accumulation efficiency with the prescriptions of Hachisu et al. (1999) and Kato \& Hachisu (2004) for $\mathrm{H}$ and He burning. These prescriptions have been widely used in the population synthesis study of type Ia supernovae (e.g., Han \& Podsiadlowski 2004; Wang et al. 2009; Liu et al. 2018).

We here simply assumed an NS birth mass of $1.4 M_{\odot}$ for convenience. However, we recall that the NS mass from different formation scenarios may be different. The mass of NSs produced by ECSNe and AIC is suggested to range from $1.25 M_{\odot}$ to $1.27 M_{\odot}$ (Timmes et al. 1996; Schwab et al. 2010; van den Heuvel 2011). Dessart et al. (2006) demonstrated that the AIC can form NSs with a mass of $\sim 1.4 M_{\odot}$. Hüdepohl et al. (2010) and Fischer et al. (2010) proposed $1.366 M_{\odot}$ and $1.347 M_{\odot}$ as the final baryon mass of NSs from the $8.8 M_{\odot}$ ECSNe progenitor, respectively. Based on the distribution of NS masses in observations, most NS masses are concentrated near $\sim 1.4 M_{\odot}($ Lattimer 2012).

The NS natal kick is crucial for the formation of binaries with NS companions because most binaries may be destroyed by $\mathrm{SNe}$ when NSs are born. Even when binaries survive the SNe, their orbital periods and eccentricities are still highly sensitive to NS natal kicks. It is commonly considered that NSs from ECSNe or AIC receive much weaker natal kicks than those from CCSNe (Pfahl et al. 2002; Podsiadlowski et al. 2004; Scheck et al. 2004; Dessart et al. 2006), but the uncertainty on this question is large. In our simulation, we assumed that no natal kicks were imparted onto NSs produced by ECSNe or AIC, and we adopted two prescriptions for the natal kick velocity for NSs from CCSNe.

(i) Hobbs et al. (2005) suggested a well-fit Maxwellian velocity distribution with a dispersion of $\sigma=265 \mathrm{~km} \mathrm{~s}^{-1}$ based on an analysis of proper motion measurements of a variety of pulsars,

$P\left(v_{\mathrm{k}}\right)=\sqrt{\frac{2}{\pi}} \frac{v_{\mathrm{k}}^{2}}{\sigma^{3}} \mathrm{e}^{-v_{\mathrm{k}}^{2} / 2 \sigma^{2}}$.

(ii) Inspired by $\mathrm{SN}$ explosion asymmetries, Bray \& Eldridge (2016, 2018) proposed a direct connection between the kick velocities and the ratio of the ejected mass of an $\mathrm{SN}$ explosion to NS mass, that is,

$v_{\mathrm{k}}=\alpha\left(\frac{M_{\text {eject }}}{M_{\text {remnant }}}\right)+\beta$, where $M_{\text {eject }}$ is ejecta mass from the SN, $M_{\text {remnant }}$ is the NS mass, and $\alpha$ and $\beta$ are constants. Bray \& Eldridge (2018) suggested a best-fit kick with $\alpha=100$ and $\beta=-170$. For convenience, we denote them with $V k_{\sigma=265 \mathrm{~km} \mathrm{~s}^{-1}}$ and $V k_{\text {linear }}$.

\subsection{Dynamical instability and CE evolution}

It is an unresolved problem for the binary interaction whether the mass transfer is dynamically stable. To determine the mass transfer stability, we assumed that if the mass ratio at the onset of mass transfer is higher than a critical mass ratio $q_{\mathrm{c}}$ (mass ratio of the donor to the accretor), the mass transfer is dynamically unstable and a $\mathrm{CE}$ forms soon after. We set $q_{\mathrm{c}}$ to be 3.0 for donors on the MS, He MS, and in the He-core burning phase, and 4.0 for donors during the HG. When the donors were on the GB, the model of condensed polytrope was adopted (Hjellming \& Webbink 1987), that is,

$q_{\mathrm{c}}=0.362+\left[3\left(1-M_{\mathrm{c}} / M_{\mathrm{d}}\right)\right]^{-1}$,

where $M_{\mathrm{d}}$ and $M_{\mathrm{c}}$ are the mass and core mass of the donor, respectively. In particular, $q_{\mathrm{c}}$ was set to be 0.784 for He stars in GB (see Hurley et al. 2002). Note that the $q_{\mathrm{c}}$ given here is valid for the evolution before the formation of NS+MS binaries. The following evolution of NS+MS binaries depends on the detailed binary evolution calculation as described in Sect. 2 and in Paper I.

The CE evolution is another unresolved problem in binary evolution (see Ivanova et al. 2013 for a review). We used the standard energy prescription (Webbink 1984) for the CE evolution in our study, that is,

$E_{\mathrm{b}}=\alpha_{\mathrm{CE}}\left(E_{\mathrm{o}, \mathrm{f}}-E_{\mathrm{o}, \mathrm{i}}\right)$,

where $E_{\mathrm{o}, \mathrm{f}}$ and $E_{\mathrm{o}, \mathrm{i}}$ are final and initial orbit energy, respectively. $E_{\mathrm{b}}$ is the binding energy of the $\mathrm{CE}$ and is written as

$E_{\mathrm{b}}=\frac{G M_{\mathrm{d}} M_{\mathrm{e}}}{\lambda R_{\mathrm{d}}}$,

where $M_{\mathrm{e}}$ and $R_{\mathrm{d}}$ are the envelope mass and radius of the donor, respectively. The CE ejection efficiency $\alpha_{\mathrm{CE}}$ and structure parameter of the envelope $\lambda$ are highly uncertain.

In our simulations, the $\lambda$ value was computed with the fitting formula of Claeys et al. (2014; see their Appendix A), where $\lambda$ varies with stellar mass, envelope mass, luminosity, and evolutionary stage (i.e., stellar type). The $\alpha_{\mathrm{CE}}$ value was assumed to be a constant and was set it to be $0.5,1,2$, and 3 . Being a measure of energy conversion efficiency, values of alpha that are higher than 1 are technically unphysical. However, because other sources of energy in the envelope are not considered, models with values of alpha higher than 1, which reflect the presence of these additional energy sources (see, e.g., Fragos et al. 2019), tend to perform better in general when tested by observations.

\section{Results}

\subsection{Sample of $N S+M S, N S+H G$, and $N S+G B$ binaries}

In our simulations, we first obtained a sample of $\mathrm{NS}+\mathrm{MS}$, $\mathrm{NS}+\mathrm{HG}$, and $\mathrm{NS}+\mathrm{GB}$ binaries from BSE, then obtained the $\mathrm{sdB}+\mathrm{NS}$ binaries by interpolating in the model grid. The properties of $\mathrm{NS}+\mathrm{MS}$, NS+HG, and $\mathrm{NS}+\mathrm{GB}$ binaries are therefore helpful for understanding the formation process of $\mathrm{sdB}+\mathrm{NS}$ binaries. 

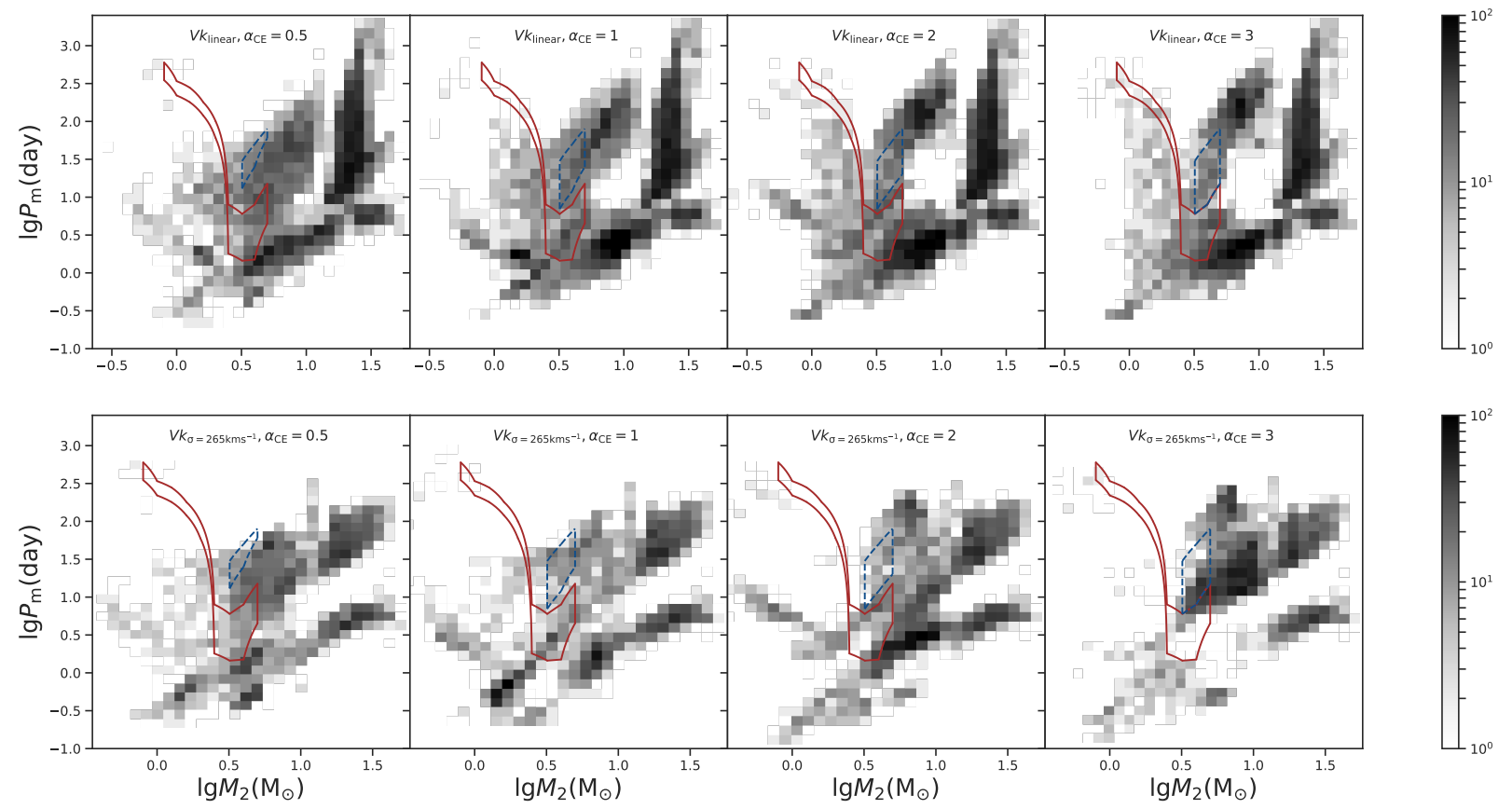

Fig. 3. Number density distribution of NS+MS, NS+HG, and NS+GB binaries on the mass $\left(\log M_{2}\right)-$ period $\left(\log P_{\mathrm{m}}\right)$ plane when the MS, HG, and GB companion just fills its Roche lobe. The regions enclosed within the solid and dashed lines are parameter spaces for producing sdB+NS binaries from the RLOF channel and from the CE ejection channel, respectively. The prescriptions for NS kicks and the value of $\alpha_{\mathrm{CE}}$ are indicated in each panel.

\subsubsection{Distribution of the companion mass - orbit period}

In Fig. 3 we present the distribution of $\mathrm{NS}+\mathrm{MS}, \mathrm{NS}+\mathrm{HG}$, and NS+GB binaries in the $\log M_{2}-\log P_{\mathrm{m}}$ plane, where $P_{\mathrm{m}}$ is the orbital period when the companion just fills its Roche lobe. The parameter spaces for producing $\mathrm{sdB}+\mathrm{NS}$ binaries are overplotted for comparison.

In all the simulation sets, $\mathrm{NS}+\mathrm{MS}, \mathrm{NS}+\mathrm{HG}$, and $\mathrm{NS}+\mathrm{GB}$ binaries are from two evolutionary channels. Binaries with more massive companions $\left(\log M_{2} \geq 1.1\right)$ are from the stable RLOF channel, and the $\mathrm{CE}$ ejection channel results in less massive companions $\left(\log M_{2}<1.1\right)$. The mass of $M_{2}$ from the stable RLOF channel is so massive because they are initially massive to ensure that the mass transfer is stable and that they accrete some material during RLOF. Figure 3 shows that all the NS+MS, $\mathrm{NS}+\mathrm{HG}$, and NS+GB binaries from stable RLOF are outside the parameter space and do not contribute to the production of sdB+NS binaries. Very few $\mathrm{NS}+\mathrm{MS}, \mathrm{NS}+\mathrm{HG}$, and $\mathrm{NS}+\mathrm{GB}$ binaries (especially in the bottom panels) fall in the parameter space for producing sdB+NS binaries when $\log M_{2}<0.4$ $\left(M_{2}<2.0 M_{\odot}\right)$. This is expected because NSs with such lowmass MS, HG, and GB components are from $\mathrm{CE}$ ejection and therefore have relatively short orbital periods, while long orbital periods are required to produce $\mathrm{sdB}$ stars from such low-mass progenitors. Even if He star+MS binaries with a long orbital period could be produced after CE ejection, these systems are likely to be destroyed during an SN if the NS natal kick is strong enough, such as the $V k_{\sigma=265 \mathrm{~km} \mathrm{~s}^{-1}}$ case shown in the bottom panels of Fig. 3. This significantly affects all properties of $s \mathrm{~dB}+\mathrm{NS}$ binaries, as we show below.

\subsubsection{Effect of $\alpha_{\mathrm{CE}}$}

In binary evolution, the orbital period $P_{\mathrm{m}}$ of an NS+MS, $\mathrm{NS}+\mathrm{HG}$, and $\mathrm{NS}+\mathrm{GB}$ binary produced from $\mathrm{CE}$ ejection is affected by two factors: the value of $\alpha_{\mathrm{CE}}$, and the NS natal kicks. The higher the $\alpha_{\mathrm{CE}}$ value, the longer the orbital period of $\mathrm{He}$ star+MS binaries after the $\mathrm{CE}$ ejection. These $\mathrm{He}+\mathrm{MS}$ binaries are likely destroyed by $\mathrm{SN}$ in the subsequent evolution. In the case of $V k_{\sigma=265 \mathrm{~km} \mathrm{~s}^{-1}}$, the NS natal kick is independent of the He star mass. The distribution on the $\log M_{2}-\log P_{\mathrm{m}}$ plane then changes significantly for the different $\alpha_{\mathrm{CE}}$ values, that is to say, more systems have long $P_{\mathrm{m}}$ with increasing $\alpha_{\mathrm{CE}}$. For the $V k_{\text {linear }}$ description, however, the NS natal kick depends on the He star mass. The effect from different $\alpha_{\mathrm{CE}}$ values is offset by NS kick velocities, and the distribution changes little, as shown in the top panels of Fig. 3.

\subsubsection{Effects of NS kicks}

The prescription of NS natal kick has a great effect on the formation of NS+MS, NS+HG, and NS+GB binaries. The higher kick velocity may result in more binaries being destroyed in $\mathrm{SN}$ explosion and in higher eccentricities of orbits after SN explosion if the binaries have not been destroyed.

As shown in the bottom three panels of Fig. $3\left(V k_{\sigma=265 \mathrm{~km} \mathrm{~s}^{-1}}\right)$, there is an obvious gap for the orbital periods when $\log M_{2} \geq$ 1.1. This is due to different strengths of the tidal effect on binaries with different orbital periods after SN explosions. When binaries have short orbital periods after SN explosion, a strong tidal effect causes the orbit to become circular soon, and the secondary fills its Roche lobe as usual. This corresponds to the NS+MS, NS+HG, and NS+GB binaries below the gap in the figure. However, for binaries with intermediate orbital periods after SN explosions, the tidal effect is not strong enough and the orbits maintain high eccentricities for a long time. The binary separation of binaries at periastron is smaller than the Roche-lobe radius of the secondary (In the BSE code, the Rochelobe radius is calculated with the following formula: $\frac{R_{\mathrm{rl}}}{a}=$ 


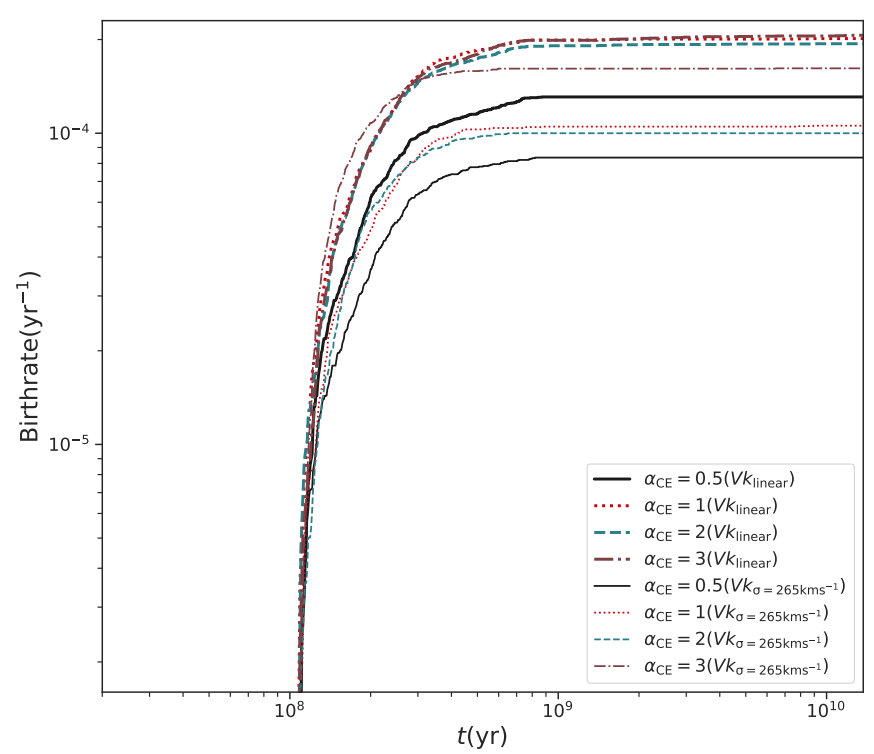

Fig. 4. Birthrates of $s d B+N S$ binaries in our simulations when a constant Galactic star formation rate of $5 M_{\odot} \mathrm{yr}^{-1}$ is assumed. Different line styles represent different parameter settings of the simulation.

$0.49 q^{2 / 3}$ $\frac{0.49 q^{2 / 3}}{0.6 q^{2 / 3}+\ln \left(1+q^{1 / 3}\right)}$, where $a$ is the semi-major axis instead of the binary separation in an eccentric orbit, and $q$ is the binary mass ratio). As the secondary evolves, its radius increases and engulfs the NS first at periastron before it fills its Roche lobe. In this case, the NS+MS, NS+HG, and NS+GB binaries most likely enter the CE phase and eventually merge. Therefore they do not appear in the figure. Furthermore, for those with long orbital periods after $\mathrm{SN}$ explosions, the Roche-lobe radius of the secondary is smaller than the binary separation at periastron. The MS, HG, and GB companions will fill their Roche lobe first. These binaries correspond to the part above the gap in the future.

The gap disappears in models with the $V k_{\text {linear }}$ description (the top three panels in Fig. 3). In this case, the kick velocity imparted on the NS is significantly weaker than that from the $V k_{\sigma=265 \mathrm{~km} \mathrm{~s}^{-1}}$ description when $\log M_{2} \geq 1.1$. Then the eccentricities accordingly decrease, which avoids the merger of NS+MS, $\mathrm{NS}+\mathrm{HG}$, and NS+GB binaries at periastron.

In the $V k_{\text {linear }}$ description when $0.7<\log M_{2}<1.1$, another gap occurs. The NS+MS, NS+HG, and NS+GB binaries in this range stem from the $\mathrm{CE}$ ejection channel. The progenitors of NSs are more massive than those from the stable RLOF with $\log M_{2}>1.1$. The ejected mass during the $\mathrm{SNe}$ is therefore relatively high and the NSs receives much higher kick velocities, according to the $V k_{\text {linear }}$ description. For similar reasons as described above, the NS+MS, NS+HG, and NS+GB binaries with intermediate orbital periods might merge at periastron, leaving the gap in this range.

\subsection{Properties of Galactic $s d B+N S$ binaries}

\subsubsection{Numbers and birth rates}

In order to obtain the birthrates and numbers of $\mathrm{sdB}+\mathrm{NS}$ binaries of the Galaxy from different simulation sets, we first studied the case of a single starburst, then convolved the binaries with the star formation history of the Galaxy. Here we adopted a constant star formation rate of $5 M_{\odot} \mathrm{yr}^{-1}$ (see Willems \& Kolb 2004). When we count the numbers of $\mathrm{sdB}+\mathrm{NS}$ binaries at the present epoch in the Galaxy, we need to know the lifetime of an $\mathrm{sdB}+\mathrm{NS}$ binary, $t_{\mathrm{sdB}+\mathrm{NS}}$. In general, the value of $t_{\mathrm{sdB}+\mathrm{NS}}$ is taken as the time of the core He burning of the sdB. However, if the merger time from GWR, $t_{\text {merge }}$, is shorter than the timescale for core He burning, we adopt $t_{\text {merge }}$ as the $t_{\mathrm{sdB}+\mathrm{NS}}$.

Figure 4 shows the birthrate as a function of age for our Galaxy. The birthrate sharply increases when the age is just older than $10^{8} \mathrm{yr}$, and it is constant after about $(5-6) \times 10^{8} \mathrm{yr}$. This property is mainly determined by the evolutionary age of the progenitors of sdB stars.

The current birthrate of $\mathrm{sdB}+\mathrm{NS}$ binaries in the Galaxy is about $10^{-4} \mathrm{yr}^{-1}$, and the number is $\sim 7000-21000$, a large portion of which are from the CE+RLOF channel. The CE+CE channel only contributes little to Galactic sdB+NS binaries because the lifetimes of $\mathrm{sdB}+\mathrm{NS}$ binaries in this way are too short, that is, they live some megayears (see Paper I). With the increase in $\alpha_{\mathrm{CE}}$, the orbital periods after CE ejection increase accordingly, and the merger timescale tends to be longer. Thus the portion of $\mathrm{sdB}+\mathrm{NS}$ binaries from the $\mathrm{CE}+\mathrm{CE}$ channel gradually increases. The birthrate has the highest value of $2.06 \times$ $10^{-4} \mathrm{yr}^{-1}$ for set 4 , and it changes little with $\alpha_{\mathrm{CE}}$ for a given prescription of NS natal kick.

Comparing our results with those of the comprehensive study of Han et al. (2003), we find that for set 4, the sdB+NS binaries contribute $0.3-0.5 \%$ to the total number of $\mathrm{sdB}$ binaries. The number differences induced by different prescriptions for NS natal kicks are within a factor of $\sim 2$, and the linear formula of kick velocity considering the effect of various He star masses before SN results in higher birthrates.

Our study shows that the fraction of $\mathrm{sdB}+\mathrm{NS}$ binaries from the ECSNe and AIC channel varies with $\alpha_{\mathrm{CE}}$. This fraction is about $22-37 \%, 35-73 \%, 18-40 \%$, and $6-19 \%$ for the models with $\alpha_{\mathrm{CE}}=0.5,1,2$, and 3 , respectively. In the model with $\alpha_{\mathrm{CE}}=1$, the typical orbital period of $\mathrm{NS}+\mathrm{MS}, \mathrm{NS}+\mathrm{HG}$, and $\mathrm{NS}+\mathrm{GB}$ binaries from the ECSNe and AIC channel is about 0.5-10 days. Most of these binaries are located in the parameter space that is optimal for producing $\mathrm{sdB}+\mathrm{NS}$ binaries. With any change of $\alpha_{\mathrm{CE}}$ relative to this value, the orbital periods of $\mathrm{NS}+\mathrm{MS}, \mathrm{NS}+\mathrm{HG}$, and $\mathrm{NS}+\mathrm{GB}$ binaries from the ECSNe and AIC channel changes in a way that increasingly more binaries are located outside of the optimal area in parameter space. The number of $\mathrm{sdB}+\mathrm{NS}$ binaries emanating from the ECSNe and AIC channel will therefore decrease with any deviation of the $\alpha_{\mathrm{CE}}$ value from 1 . The properties of these binaries, such as delay time, mass of the sdBs, and orbital periods, cannot be distinguished from that of the $\mathrm{sdB}+\mathrm{NS}$ binaries that experienced the CCSNe because the properties of $\mathrm{sdB}+\mathrm{NS}$ binaries are mainly determined by the progenitors for $\mathrm{sdB}$ stars.

\subsubsection{The sdB mass, orbital period, and RV semi-amplitude}

While the $\alpha_{\mathrm{CE}}$ value has little effect on the birthrate and number of Galactic sdB+NS binaries, it affects the orbital periods of the $\mathrm{sdB}+\mathrm{NS}$ binaries. Figure 5 shows the sdB mass - period $\left(M_{\mathrm{sdB}}-\log P\right)$ distribution of Galactic $\mathrm{sdB}+\mathrm{NS}$ binaries at the current epoch from our simulations. With the exception of the models with $\alpha_{\mathrm{CE}}=0.5$, we obtain two groups of $\mathrm{sdB}+\mathrm{NS}$ binaries for each simulation set: one (with long $P$, from several to $\sim 1000$ d) from the CE+RLOF channel, and the other (with short $P$, shorter than $\sim 0.1 \mathrm{~d}$ ) from the $\mathrm{CE}+\mathrm{CE}$ channel. The distributions are very similar to each other in the upper panels (for $\left.V k_{\text {linear }}\right)$, but display a certain level of divergence among the lower ones (for $V k_{\sigma=265 \mathrm{~km} \mathrm{~s}^{-1}}$ ). The models with $\alpha_{\mathrm{CE}}=0.5$ are an exception because these models only generate $\mathrm{sdB}+\mathrm{NS}$ binaries that can currently be observed through the CE+RLOF channel. The greatest difference comes from set 8 , for which the samples 


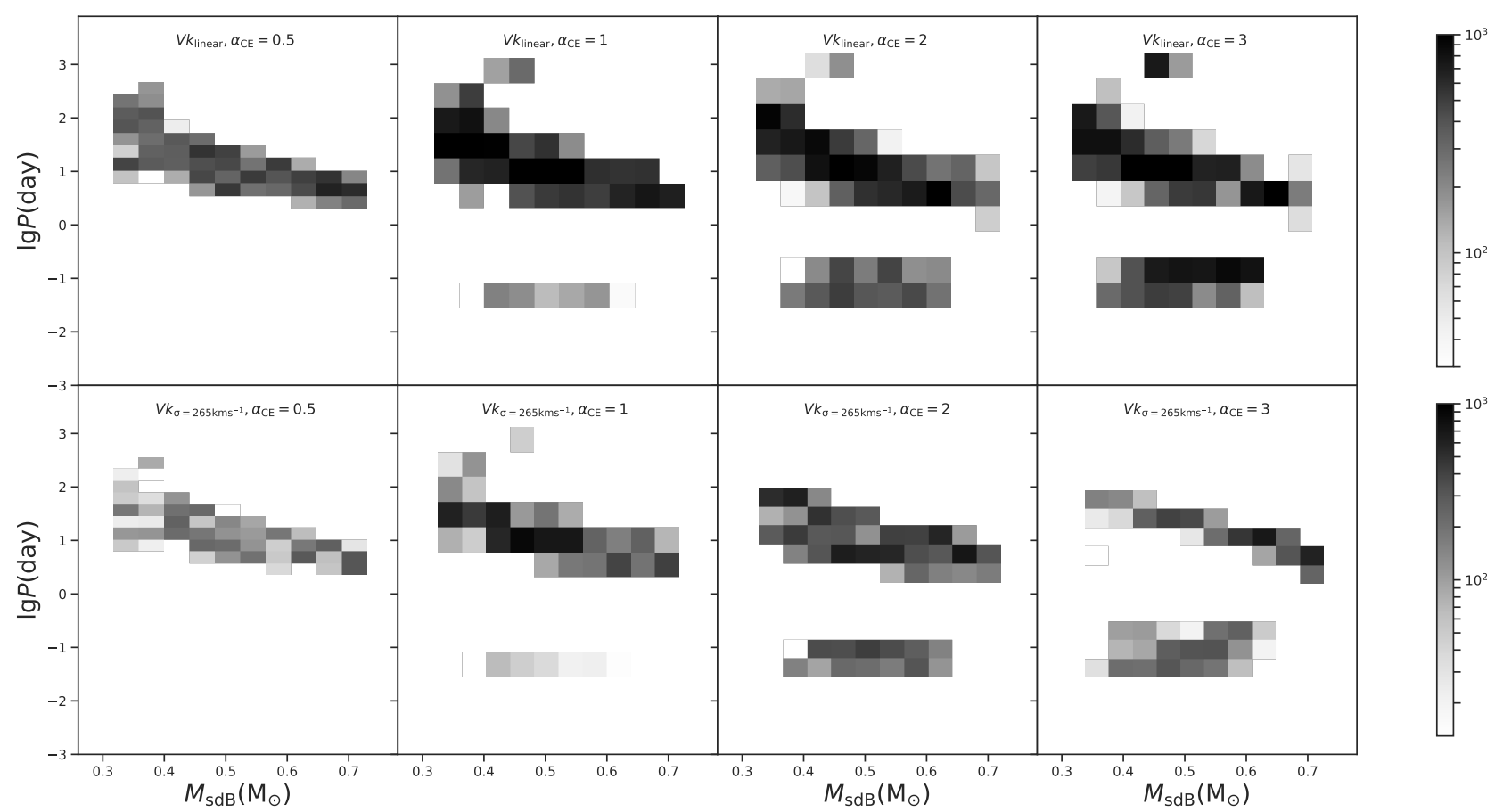

Fig. 5. Number distributions in the $\mathrm{sdB}$ mass $\left(M_{\mathrm{sdB}}\right)$ - period $(\log P)$ diagram for Galactic sdB $+\mathrm{NS}$ binaries at the present epoch. The gray scale shows the numbers of each pixel, and the parameter settings for the simulation are indicated in each panel.

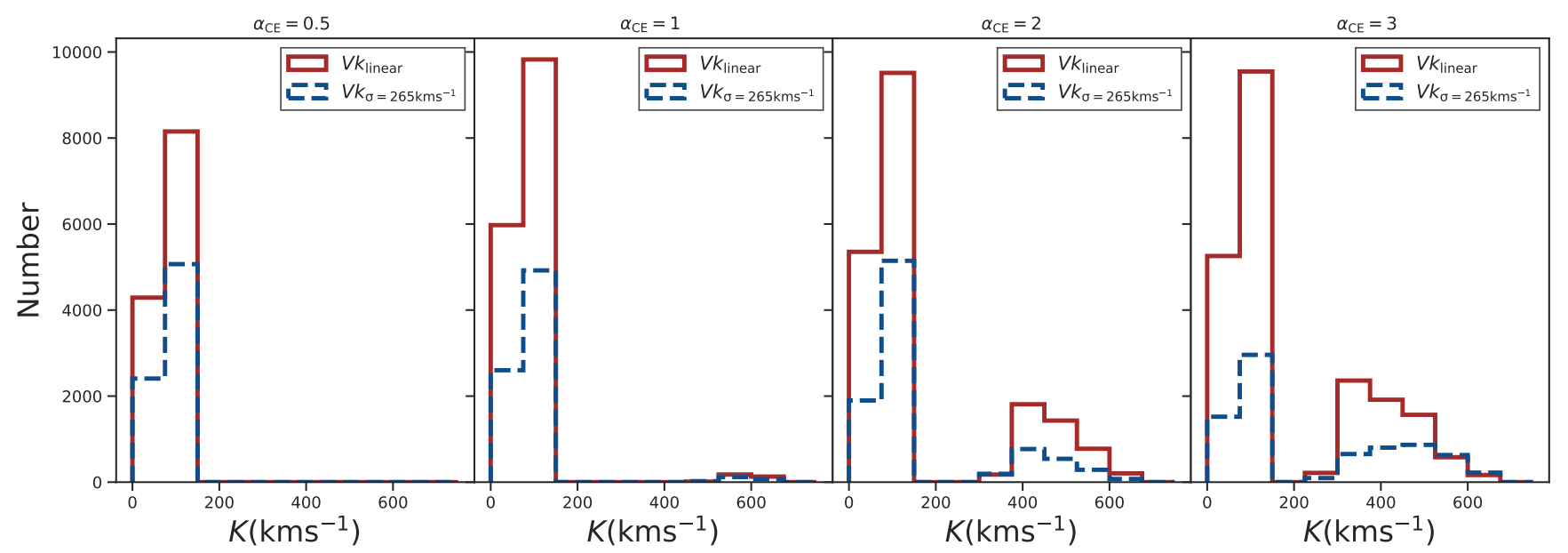

Fig. 6. Radial velocity semi-amplitude $K$ distributions of Galactic sdB+NS binaries at the present epoch. The value of $\alpha_{\mathrm{CE}}$ is described at the top. The prescriptions of NS natal kicks, i.e., $V k_{\text {linear }}\left(\right.$ red) and $V k_{\sigma=265 \mathrm{~km} \mathrm{~s}^{-1}}$ (blue) are indicated in the legend.

from the CE+RLOF channel obviously gather at the long-period end. This can be understood as follows. Because of the relatively high $\alpha_{\mathrm{CE}}$ value for set 8 (in comparison to sets 5,6 , and 7), the NS+MS, NS+HG, and NS+GB binaries produced by the primary binary evolution (the first binary interaction) have long periods in general, as shown in the bottom right panel of Fig. 3. This leads to relatively long periods for the produced $\mathrm{sdB}+\mathrm{NS}$ binaries from the CE+RLOF channel in the following evolution, and thus a distribution that is more highly concentrated at the long-period end.

There is a wide range of sdB masses, $\sim 0.32-0.75 M_{\odot}$, but without a clear mass peak, as shown in Fig. 5. The sdBs from the $\mathrm{CE}$ channel have a narrower mass range, but the distribution still does not show a peak. The sdB+NS binaries from the CE+RLOF channel do not agree with the sdB mass - period relation given by Chen et al. (2013) because the progenitors for sdB stars are more massive than $2.5 M_{\odot}$ (therefore have non-degenerate He cores) in general, as shown in Fig. 3.

Figure 6 shows the RV semi-amplitude $K$ distribution for Galactic sdB+NS binaries, where the value of $K$ is derived from the binary mass function (e.g., Geier et al. 2011) by assuming an inclination angle of $90^{\circ}$. The main peak arises at about $150 \mathrm{~km} \mathrm{~s}^{-1}$ for the samples from the CE+RLOF channel, and a small peak lies between 400 and $600 \mathrm{~km} \mathrm{~s}^{-1}$, produced from the $\mathrm{CE}+\mathrm{CE}$ channel. Again, $\alpha_{\mathrm{CE}}=0.5$ leads to exceptions, for the same reason as explained above.

\subsubsection{Progenitor ages}

In addition to the characteristics of $\mathrm{sdB}+\mathrm{NS}$ binaries, the progenitors also hold some clues for searching them. Figure 3 has already shown that $M_{2}$, which is more massive than $2.5 M_{\odot}$, 


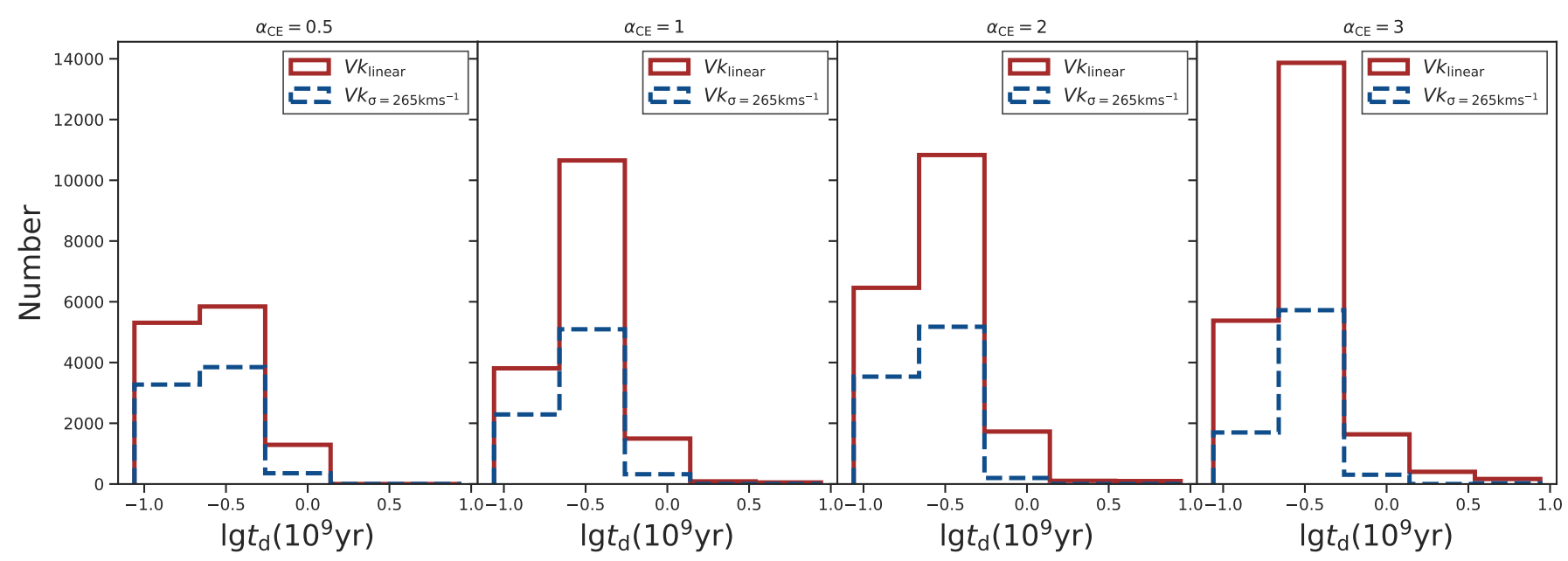

Fig. 7. Similar to Fig. 6, but for the delay time $t_{\mathrm{d}}$ (from the primordial zero-age main-sequence binaries to the formation of sdB $+\mathrm{NS}$ binaries) distributions of Galactic $\mathrm{sdB}+\mathrm{NS}$ binaries at the present epoch.

can generally produce $\mathrm{sdB}+\mathrm{NS}$ binaries from the $\mathrm{CE}+\mathrm{RLOF}$ channel or the $\mathrm{CE}+\mathrm{CE}$ channel. For each $\mathrm{sdB}+\mathrm{NS}$ binary in the Galaxy, we determined the delay time $t_{\mathrm{d}}$ (i.e., from the primordial zeor-age main-sequence binaries to the formation of $\mathrm{sdB}+\mathrm{NS}$ binaries) and show its distribution in Fig. 7. The peak around $0.2 \mathrm{Gyr}$ is obvious, and the majority has delay times shorter than $10^{9} \mathrm{yr}$. This indicates that Galactic sdB+NS binaries are born in very young populations, which supports the idea that $\mathrm{sdB}+\mathrm{NS}$ binaries are located in the Galactic disk. The fact that no $\mathrm{sdB}+\mathrm{NS}$ binaries have been discovered by the MUCHFUSS project is then easily understood because the candidates for the project are from the SDSS, and most objects exceeding $\sim 3 \mathrm{kpc}$ may be located in the Galactic halo and are old.

\subsection{GWR signals from short-period $s d B+N S$ binaries}

As noted in Paper I, the sdB+NS binaries produced from the $\mathrm{CE}+\mathrm{CE}$ channel could be identified as gravitational wave radiation (GWR) sources because their orbital periods are extremely short. Our simulations show that up to $\sim 6000 \mathrm{sdB}+\mathrm{NS}$ binaries can be produced from the $\mathrm{CE}+\mathrm{CE}$ channel at the present epoch in the Galaxy (see Table 2), depending on the values of $\alpha_{\mathrm{CE}}$. The GWR characteristic strains $h_{\mathrm{c}}$ versus frequency $f$ for these objects are presented in Fig. 8. The distance for each sample was obtained through a Monte Carlo simulation with the Galactic potential model of Astraatmadja \& Bailer-Jones (2016), similar to what has been done in Yu \& Jeffery (2010).

Figure 8 shows that part of these $\mathrm{sdB}+\mathrm{NS}$ binaries $(\sim 100-300)$ radiate $\mathrm{GW}$ with strains above the sensitivity curve of the Laser Interferometer Space Antenna (LISA) for an $S / N=$ 1. Although the signals may be covered by confusion-limited background at low frequencies (The confusion-limited noise background can be produced from double white dwarfs at low frequencies $\sim \lg f<-2.8$ see Nelemans et al. 2001), systems with a very strong signal might be extracted from the noise (Littenberg 2011). They may be detectable by LISA in the future.

\section{Conclusions and outlook}

The purpose of this study was to obtain the population properties of Galactic sdB+NS binaries through BPS studies, such as the number (or birthrate), orbital period, RV semi-amplitude distributions, and the delay times. Different prescriptions of NS
Table 2. Results for various models.

\begin{tabular}{|c|c|c|c|c|}
\hline Set & Number $^{(a)}$ & $\mathrm{CE}+\mathrm{CE}^{(b)}$ & $\mathrm{CE}+\mathrm{RLOF}^{(c)}$ & $\begin{array}{l}\text { Birthrate } \\
\left(10^{-4} \mathrm{yr}^{-1}\right) \\
\end{array}$ \\
\hline 1 & 12491 & $0 \%$ & $100 \%$ & 1.31 \\
\hline 2 & 16120 & $2 \%$ & $98 \%$ & 2.01 \\
\hline 3 & 19265 & $23 \%$ & $77 \%$ & 1.94 \\
\hline 4 & 21604 & $30 \%$ & $70 \%$ & 2.06 \\
\hline 5 & 7510 & $0 \%$ & $100 \%$ & 0.83 \\
\hline 6 & 7733 & $3 \%$ & $97 \%$ & 1.06 \\
\hline 7 & 8910 & $21 \%$ & $79 \%$ & 1.00 \\
\hline 8 & 7748 & $40 \%$ & $60 \%$ & 1.62 \\
\hline
\end{tabular}

Notes. ${ }^{(a)}$ Total estimated number in the Galaxy at the present epoch; ${ }^{(b)}$ percentages of $\mathrm{sdB}+\mathrm{NS}$ binaries from the $\mathrm{CE}+\mathrm{CE}$ channel of the total number; ${ }^{(c)}$ percentages of $\mathrm{sdB}+\mathrm{NS}$ binaries from the $\mathrm{CE}+\mathrm{RLOF}$ channel of the total number; ${ }^{(d)}$ birthrate of $\mathrm{sdB}+\mathrm{NS}$ binaries at the present epoch.

natal kicks and parameters of CE evolution were investigated to evaluate the uncertainties from SN explosion and from binary evolution. Our main conclusions are listed below.

(i) There are two mass-transfer processes from a primordial binary that evolves into an $\mathrm{sdB}+\mathrm{NS}$ binary. The first process (for producing a He star, then an NS) is dynamically unstable. The second mass-transfer process might be dynamically stable or unstable, and the low-mass MS, HG, andGB companions ( $\leq 2.0 M_{\odot}$, with degenerate He cores) hardly contribute to the formation of sdB+NS binaries because the orbital periods of NS binaries with such low-mass companions are not long enough for the companion to evolve into sdBs.

(ii) The Galactic birthrate of $\mathrm{sdB}+\mathrm{NS}$ binaries is about $10^{-4} \mathrm{yr}^{-1}$, and the highest value is $2.06 \times 10^{-4} \mathrm{yr}^{-1}$ in our simulations (from set 4 ). There are $\sim 7000-21000 \mathrm{sdB}+\mathrm{NS}$ binaries in the Galaxy at the present epoch, which contributes $0.3-0.5 \%$ of the total sdB binaries for set 4 . The parameters describing the CE evolution have little impact on the results, while the prescription for NS natal kicks affects the number (and birthrate) by a factor of $\sim 2$. The prescription that considers the effect of the progenitor mass of NS results in higher birthrates.

(iii) Most of Galactic sdB+NS binaries $(\gtrsim 60 \%)$ stem from the CE+RLOF channel. The populations from the CE+RLOF 


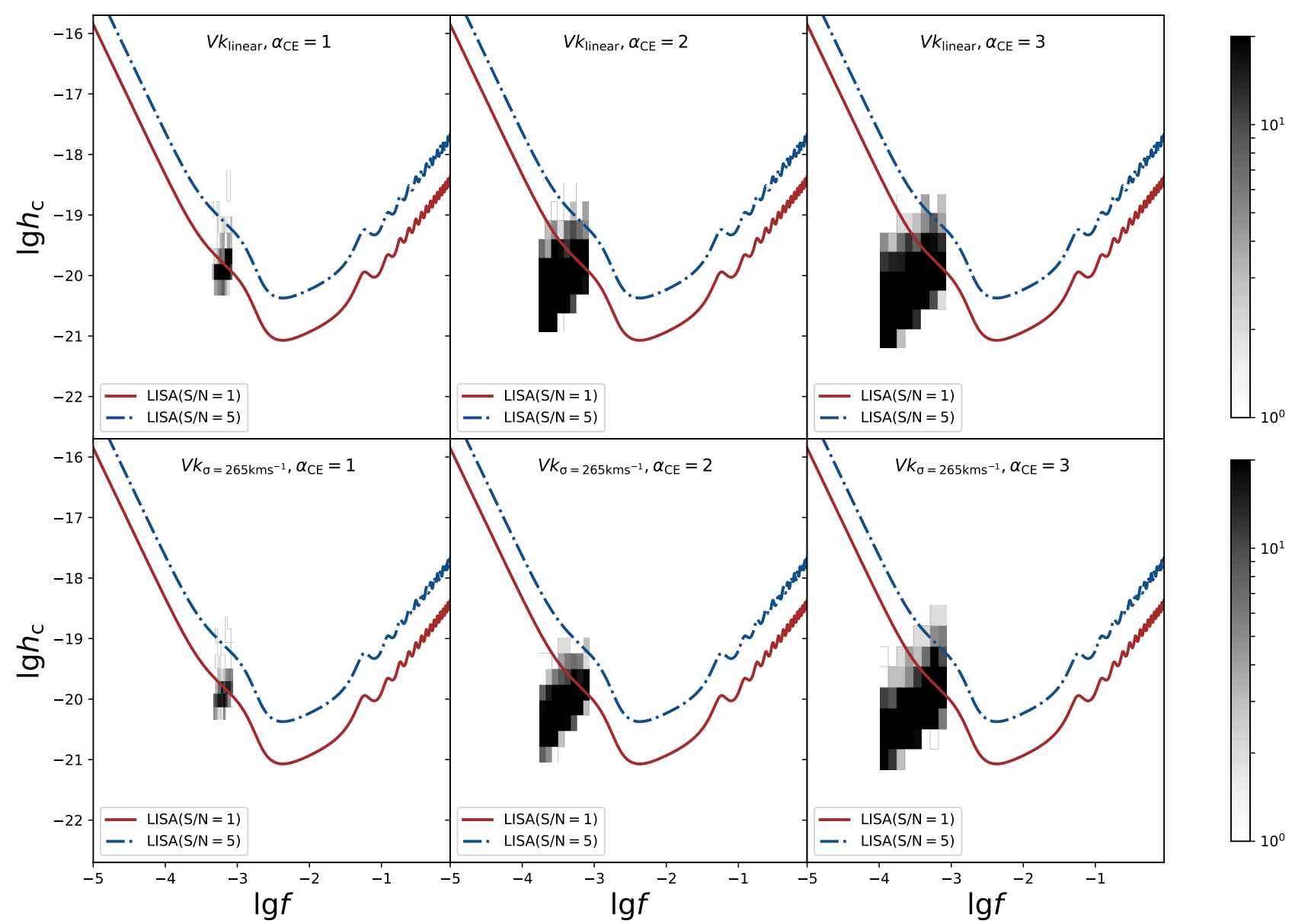

Fig. 8. Gravitational wave characteristic strain vs. frequency sdB+NS binaries from the CE+CE ejection channel in the Galaxy. The solid and dot-dashed lines are LISA sensitivity curves for an S/N of 1 and 5, respectively, with an integration time of 4 yr (Klein et al. 2016).

channel and from the $\mathrm{CE}+\mathrm{CE}$ channel show different orbital periods, as expected. This results in two peaks in the RV semi-amplitude distribution: $100-150 \mathrm{~km} \mathrm{~s}^{-1}$ for those from CE+RLOF channel, and $400-600 \mathrm{~km} \mathrm{~s}^{-1}$ for those from the $\mathrm{CE}+\mathrm{CE}$ channel. However, the delay times for the two populations are similar, that is, they have a peak at about $0.2 \mathrm{Gyr}$ and are no older than 1.0 Gyr. This indicates that Galactic sdB+NS binaries are born in very young populations. We therefore suggest that most $\mathrm{sdB}+\mathrm{NS}$ binaries may be located in the Galactic disk and have a small RV semi-amplitude. The observation of the MUCHFUSS project can be easily understood. It might still be difficult to discover the binaries in the Galactic disk because the $K$ values are low and the extinction is high.

(iv) We find an upper limit of $\sim 6000$ for the number of $\mathrm{sdB}+\mathrm{NS}$ binaries that evolved through the $\mathrm{CE}+\mathrm{CE}$ channel in the Galaxy. They are potentially strong GWR sources. About $\sim 100-300$ might be detected by LISA 4 yr observations with an $S / N=1$.

The results for $\mathrm{sdB}+\mathrm{NS}$ binaries have several important implications for future studies. For instance, during the formation of the sdB+NS binaries, the NS can be spun up to be a millisecond pulsar from the stable RLOF. Moreover, carbon-oxygen ultra-compact X-ray binary might be formed from $\mathrm{sdB}+\mathrm{NS}$ binaries with short orbital periods. Further studies regarding the subsequent evolution phase of $\mathrm{sdB}+\mathrm{NS}$ binaries are warranted.

Acknowledgements. This work is partly supported by the Natural Science Foundation of China (Nos. 11733008, 11521303, 11703081), the National
Ten-thousand talents program, CAS light of West China Program and the Youth Innovation Promotion Association of the CAS (grant 2018076).

\section{References}

Astraatmadja, T. L., \& Bailer-Jones, C. A. L. 2016, ApJ, 832, 137

Bray, J. C., \& Eldridge, J. J. 2016, MNRAS, 461, 3747

Bray, J. C., \& Eldridge, J. J. 2018, MNRAS, 480, 5657

Brown, T. M., Bowers, C. W., Kimble, R. A., Sweigart, A. V., \& Ferguson, H. C. 2000, ApJ, 532, 308

Charpinet, S., Fontaine, G., Brassard, P., \& Dorman, B. 1996, ApJ, 471, L103

Charpinet, S., Fontaine, G., Brassard, P., et al. 1997, ApJ, 483, L123

Chen, X., Han, Z., Deca, J., \& Podsiadlowski, P. 2013, MNRAS, 434, 186

Chruslinska, M., Belczynski, K., Klencki, J., \& Benacquista, M. 2018, MNRAS, 474, 2937

Claeys, J. S. W., Pols, O. R., Izzard, R. G., Vink, J., \& Verbunt, F. W. M. 2014, A\&A, 563, A83

Deca, J., Marsh, T. R., Østensen, R. H., et al. 2012, MNRAS, 421, 2798

Dessart, L., Burrows, A., Ott, C. D., et al. 2006, ApJ, 644, 1063

Eggleton, P. P., Fitchett, M. J., \& Tout, C. A. 1989, ApJ, 347, 998

Fischer, T., Whitehouse, S. C., Mezzacappa, A., Thielemann, F.-K., \& Liebendörfer, M. 2010, A\&A, 517, A80

Fragos, T., Andrews, J. J., Ramirez-Ruiz, E., et al. 2019, ApJ, 883, L45

Geier, S., Heber, U., Podsiadlowski, P., et al. 2010, A\&A, 519, A25

Geier, S., Heber, U., Tillich, A., et al. 2011, in American Institute of Physics Conference Series, eds. S. Schuh, H. Drechsel, \& U. Heber, 1331, 163

Geier, S., Kupfer, T., Heber, U., et al. 2015, A\&A, 577, A26

Geier, S., Kupfer, T., Heber, U., et al. 2017, A\&A, 602, C2

Hachisu, I., Kato, M., \& Nomoto, K. 1996, ApJ, 470, L97

Hachisu, I., Kato, M., \& Nomoto, K. 1999, ApJ, 522, 487

Han, Z. 1998, MNRAS, 296, 1019

Han, Z., \& Podsiadlowski, P. 2004, MNRAS, 350, 1301

Han, Z., Podsiadlowski, P., Maxted, P. L. F., Marsh, T. R., \& Ivanova, N. 2002, MNRAS, 336, 449 
Han, Z., Podsiadlowski, P., Maxted, P. F. L., \& Marsh, T. R. 2003, MNRAS, 341, 669

Han, Z., Podsiadlowski, P., \& Lynas-Gray, A. E. 2007, MNRAS, 380, 1098 Heber, U. 1986, A\&A, 155, 33

Heber, U. 2016, PASP, 128, 082001

Hjellming, M. S., \& Webbink, R. F. 1987, ApJ, 318, 794

Hobbs, G., Lorimer, D. R., Lyne, A. G., \& Kramer, M. 2005, MNRAS, 360, 974

Hüdepohl, L., Müller, B., Janka, H.-T., Marek, A., \& Raffelt, G. G. 2010, Phys. Rev. Lett., 104, 251101

Hurley, J. R., Pols, O. R., \& Tout, C. A. 2000, MNRAS, 315, 543

Hurley, J. R., Tout, C. A., \& Pols, O. R. 2002, MNRAS, 329, 897

Ivanova, N., Justham, S., Chen, X., et al. 2013, A\&ARv, 21, 59

Kato, M., \& Hachisu, I. 2004, ApJ, 613, L129

Kilkenny, D., Koen, C., O’Donoghue, D., \& Stobie, R. S. 1997, MNRAS, 285, 640

Klein, A., Barausse, E., Sesana, A., et al. 2016, Phys. Rev. D, 93, 024003

Lattimer, J. M. 2012, Annu. Rev. Nucl. Part. Sci., 62, 485

Littenberg, T. B. 2011, Phys. Rev. D, 84, 063009

Liu, D., Wang, B., \& Han, Z. 2018, MNRAS, 473, 5352

Maxted, P., Heber, U., Marsh, T., \& North, R. 2001, MNRAS, 326, 1391

Michel, F. C. 1987, Nature, 329, 310

Miller, G. E., \& Scalo, J. M. 1979, ApJS, 41, 513

Miyaji, S., Nomoto, K., Yokoi, K., \& Sugimoto, D. 1980, PASJ, 32, 303

Napiwotzki, R., Karl, C. A., Lisker, T., et al. 2004, Ap\&SS, 291, 321

Nelemans, G. 2010, Ap\&SS, 329, 25
Nelemans, G., Yungelson, L. R., \& Portegies Zwart, S. F. 2001, A\&A, 375, 890

Nomoto, K. 1984, ApJ, 277, 791

Nomoto, K. 1987, ApJ, 322, 206

Oppenheimer, J. R., \& Volkoff, G. M. 1939, Phys. Rev., 55, 374

Paxton, B., Bildsten, L., Dotter, A., et al. 2011, ApJS, 192, 3

Paxton, B., Cantiello, M., Arras, P., et al. 2013, ApJS, 208, 4

Paxton, B., Marchant, P., Schwab, J., et al. 2015, ApJS, 220, 15

Pfahl, E., Rappaport, S., \& Podsiadlowski, P. 2002, ApJ, 573, 283

Podsiadlowski, P., Rappaport, S., \& Pfahl, E. D. 2002, ApJ, 565, 1107

Podsiadlowski, P., Langer, N., Poelarends, A. J. T., et al. 2004, ApJ, 612, 1044

Scheck, L., Plewa, T., Janka, H.-T., Kifonidis, K., \& Müller, E. 2004, Phys. Rev. Lett., 92, 011103

Schwab, J., Podsiadlowski, P., \& Rappaport, S. 2010, ApJ, 719, 722

Tauris, T. M., Langer, N., \& Kramer, M. 2011, MNRAS

Timmes, F. X., Woosley, S. E., \& Weaver, T. A. 1996, ApJ, 457, 834

van den Heuvel, E. P. J. 2011, Bull. Astron. Soc. India, 39, 1

Vos, J., Németh, P., Vučković, M., Østensen, R., \& Parsons, S. 2018, MNRAS, 473, 693

Vos, J., Vučković, M., Chen, X., et al. 2019, MNRAS, 482, 4592

Wang, B., Meng, X., Chen, X., \& Han, Z. 2009, MNRAS, 395, 847

Webbink, R. F. 1984, ApJ, 277, 355

Willems, B., \& Kolb, U. 2004, A\&A, 419, 1057

Wu, Y, Chen, X., Li, Z, \& Han, Z, 2018, A\&A, 618, A14

Yu, S., \& Jeffery, C. S. 2010, A\&A, 521, A85 